\title{
Delays in passive and active leprosy detection in post-elimination Nepal
}

\author{
Ulla-Britt Engelbrektsson ${ }^{\mathrm{a}}$, Udaya Nath Yogi ${ }^{\mathrm{b}}$, Peter Nicholls ${ }^{\mathrm{c}} \&$ \\ Madhusudan Subedi ${ }^{\mathrm{d}}$ \\ a Associate Professor, International Nepal Fellowship, Nepal; \\ ORCID: https://orcid.org/0000-0002-4413-3695 \\ ${ }^{\mathrm{b}}$ Senior Leprosy Officer, Shining Hospital INF Surkhet, Nepal \\ ${ }^{\mathrm{c}}$ Independent Statistician, $U K$ \\ d Professor, School of Public Health, Patan Academy of Health Sciences, \\ Nepal
}

Submitted 5 January 2021; Accepted 12 January 2021

\begin{abstract}
Summary
Objectives: In late 2009, Nepal reached the leprosy elimination goal. However, the expected gradual decline in registered prevalence did not take place. In early 2015 , the International Nepal Fellowship (INF) launched a three-year research project on leprosy delays in the western part of the country. The main focus of the present article is on health-seeking processes as reported by patients diagnosed in INF outpatient skin clinics (the Outpatient cohort) and by patients diagnosed in a massive, governmentled, one-off, case detection operation (the Follow-up cohort). The former represents voluntary presentations and passive case detection, the latter, active case finding in the field.

Methods: A mixed qualitative and quantitative approach with in-depth patient interview data enhanced by consultations with key informants and on the spot observations, plus structured data from clinic records and interviews.

Results and Conclusions: The mean delay of the Outpatient cohort was found to be 28.1 months, and 23.2 months for the Follow-up cohort. While the causes of delay are complex, the long delays foremost reflect a lack of ability of individuals and families to recognize the seriousness of early leprosy symptoms compounded by health service professionals' failure to diagnose the condition.

Without early case detection and treatment, Nepal will not free itself of leprosy. A broad-scale dissemination of public education and awareness of leprosy is essential and so is an upgrading of leprosy skills among private and public health service professionals. In addition, in some contexts a double approach of self-referrals and active screening is likely to be necessary.
\end{abstract}

Keywords: Leprosy, leprosy delays, passive and active leprosy case finding, leprosy in Nepal

Correspondence to: Ulla-Britt Engelbrektsson, Associate Professor, International Nepal Fellowship, Nepal (e-mail: uengelbrektsson@inf.org) 


\section{Introduction}

In the early days of leprosy control in Nepal, case finding through large-scale population surveys was high on the agenda. However, fewer patients than anticipated were detected. Moreover, it was soon recognized that patients found by active case finding were less compliant with clinic attendance than those who had volunteered after suspecting that they might have leprosy. ${ }^{1}$ Thus, in the mid-1970s, a review of the national programme resulted in active case finding being replaced by efforts to boost voluntary presentations, the latter primarily by community health education and 'leprosy quality care'. NGOs and INGOs were deeply involved in the control work. All leprosy-related services were provided free of cost.

Multi drug therapy (MDT) was introduced in 1982. ${ }^{2}$ The number of registered leprosy cases at the time was 32,000 which corresponded to a point prevalence rate of 21 per 100,000 population.

In 1987, Nepal's previously vertical leprosy programme was formally integrated into the general health services. Seven years later, with the help of the WHO, a national plan for 'the elimination of leprosy by the year 2000' was launched. It was followed by a review which resulted in intensified and new activities, including large-scale Leprosy Elimination Campaigns. The overall results, however, were less than hoped for and the target date for achieving elimination was pushed back. Finally, in January 2010, with a reported national point prevalence rate of 0.77 per 10,000 population, the Ministry of Health announced 'the elimination of leprosy as a public health problem'. Even so, 17 districts (out of 75 at the time), had a point prevalence ratio of more than 1 per 10,000 population, most of them in the Terai belt in the south.

The expectation was that from then on there would be a gradual decline in the national leprosy prevalence. This, however, did not happen. In 2017/18 the reported national leprosy point prevalence was 0.99 per 10,000 population with registered new cases numbering 3249 (56\% multi-bacillary, $42 \%$ female, $7 \%$ children, and $4 \%$ WHO Grade 2 disability). ${ }^{3}$

To explore reasons why the expected reduction in prevalence since 2010 had not occurred, in 2015 the International Nepal Fellowship (INF) launched a three-year research project on leprosy delays in the western part of the country, the 'Delay Project'. 'Delay' was defined as "the period from a patient becoming aware of a leprosy-related symptom through to the start of leprosy treatment". In Nepal, active case finding was and is taking place but the great majority of patients present voluntarily. The aim of the project was to explore patterns of health-seeking and reasons for lengthy delays, and to look for ideas of how to shorten delays.

The Delay project, with a mixed qualitative and quantitative approach, headed by a social anthropologist, was taken forward in two of Nepal's then five Development Regions, the West and the Mid-West. Data were acquired through one-to-one interviews with patients on or about to start MDT - a patient focused approach, supplemented by register information, observations, and consultations with key informants.

In order to include patients in diverse disease and treatment circumstances, some were interviewed in their normal place of residence: 'Field patients', others after admission to hospital: 'Inpatients', and yet others were interviewed in the process of being diagnosed: 'Outpatients'. There were also two community studies primarily based upon participant observation and in-depth interviews.

The great majority of leprosy patients, as mentioned, present voluntarily. In 2017, however, there was a one-off, massive government-led active case detection operation in two districts in the south. And, a year and an half later, in 2018, as an addendum to the Outpatient study, 
data pertaining to the Active case detection operation were collected, health workers were interviewed, and so was a sample of patients diagnosed during the operation.

The present paper is the fifth in a series, the first focusing on the impact of stigma on delays - the Field-patient cohort, ${ }^{4}$ the second on the routes and challenges of health-seekingthe Inpatient cohort, ${ }^{5}$ and the third on the interplay between patients and community. ${ }^{6}$ The fourth paper is in the process of being revised. It reports on the leprosy situation and leprosy health-seeking from shortly before the start of local leprosy treatment and some forty years onwards in a rural setting in one of Nepal's hill districts. The present article focuses on health-seeking data primarily obtained through interviews with patients in the process of being diagnosed with leprosy in INF outpatient clinics in the West and Mid-west Regions (voluntary presentations). In addition, it contains information from the government-led active case-finding operation in the south including interview data from a sample of patients detected during the operation. The article provides additional data and depth to some of the key findings within the project as a whole, as well as some insights into active case finding as exemplified in the 2017 Active case detection operation in the southern districts of Banke and Bardiya.

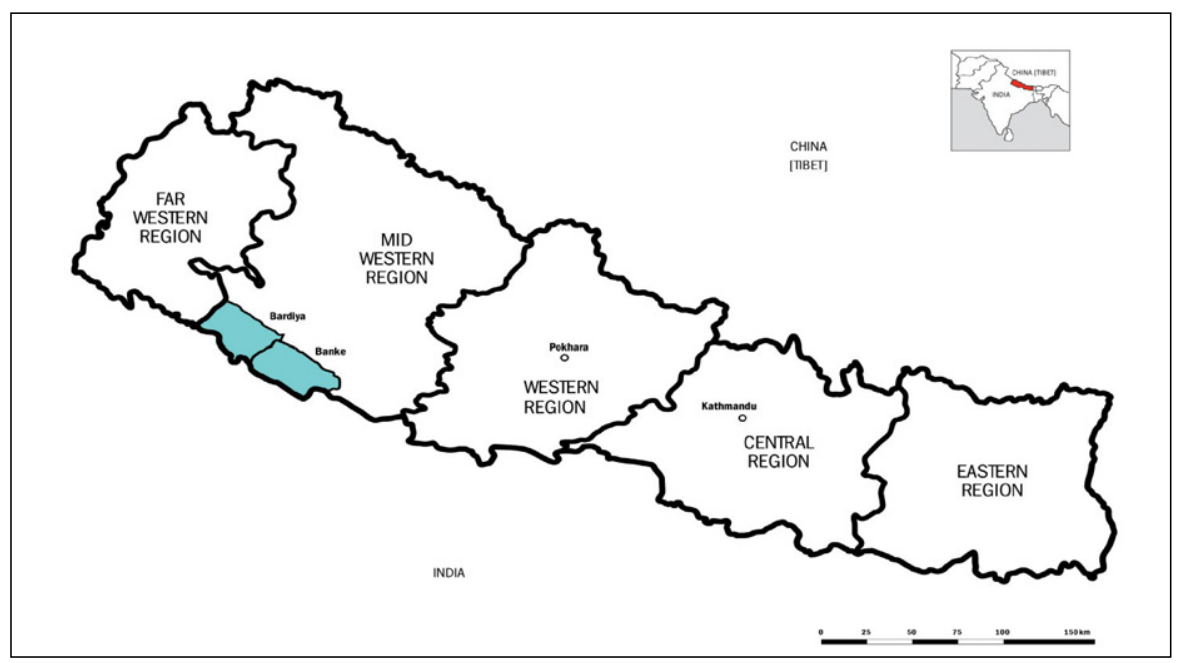

Figure 1 The west and the mid-west development regions with the districts of Banke and Bardiya highlighted.

Given the differences in sampling and location between the Outpatient and the Followup study of the Active case detection operation, the purpose of the present paper is not an immediate comparison of the two sets of data but to widen the discussion on delay to include insights into the context of active case detection. As the primary focus, the findings from Outpatient data are presented first and compared with findings from the earlier Field-patient and the Inpatient cohorts. These are followed by a separate section on findings from the Active case detection operation which was a late addition to these earlier studies and covered a wider range of themes. Remarks and Discussion and Conclusions complete the paper.

Topographically, from south to north, Nepal is divided into the Plains (the Terai), the Hills, and the Mountains; adminstratively, Nepal was divided into five regions (Figure 1). More than half of its 30 million population live in the Plains, some three million in the northern mountainous region, and the remainder in the central hilly districts. 
The infrastructure, biomedical health services included, is more developed in the south and in the cities compared with the rest of the country. At the same time, the registered leprosy prevalence rates are higher in the south than in the central and northern parts.

The Nepali therapeutic scene is pluralistic with the main building blocks being Humoral medicine (the balance of hot and cold), Ayurveda (the medical system of ancient Hinduism, including herbal medicine), magic-religious healing (mainly Shamanism), and Biomedicine, (the latecomer). ${ }^{7}$

\section{The outpatient cohort}

\section{METHODOLOGY}

From the start of the national leprosy programme in 1974, INF has been the government's main leprosy counterpart in the West and Mid-West Development Regions. INF has three hospitals with leprosy services in the two regions. There is an outpatient general skin clinic attached to each. The Outpatient cohort is a sample of patients who attended an INF skin clinic, during a twelve-month period, where they were found to be leprosy-affected. The interviews were based on an open-ended questionnaire and were carried out within hospital grounds during the waiting time between initial examination, additional tests, and diagnosis.

One reason for the Outpatient clinic approach was to ensure that the details of the healthseeking process were fresh in the minds of each patient. Another was to include as many patients as possible, the clinics rather than the field being the obvious place to meet up with multiple patients. At the same time, there was the question about the appropriateness of interviews in what could be an anxious situation for the respondents. The piloting laid such worries to rest as those approached did not hesitate to participate. Thus, during twelve months in 2016-2017, with differing start dates among the three INF centres (Shining Hospital INF Surkhet, Green Pastures Hospital Pokhara, Shining Hospital INF Banke), as many 'leprosy suspects' as circumstances allowed were interviewed. Of those interviewed, only those who received a leprosy diagnosis before the end of the day were included in the cohort. The result was a convenience sample of 218 patients, which at each centre corresponded to somewhat more than half the patients meeting the inclusion criteria, namely a Nepali citizen from the West or the Mid-West Development Region who was for the first time diagnosed as affected by leprosy.

At each centre the interviews lasted approximately half an hour, most being carried out by one and the same member of staff. The interviewers were aware of the possibility of a deference bias and context influences. They ensured that patients understood the purpose of the study; that participation was voluntary; that information obtained was confidential; and that there would be no reimbursement. They also ensured privacy of the interview situation. Less than a handful of those approached declined participation.

The main interview instrument - an open-ended questionnaire constructed to counteract recollection biases, focused on the development of signs and symptoms and on health-seeking efforts. In a roundabout way the respondent was also asked if he/she suspected or had earlier suspected 'leprosy' and if there had been leprosy among relatives. Clinical data were added after the leprosy diagnosis was confirmed.

The qualitative analysis was done by creating themes and sub-themes: signs and symptoms; the development of the disease; leprosy awareness; health-seeking actions and experiences; and ideas of what could have been done to shorten the delay. The information provided by the 
patient was supplemented by on the spot observations and clinic records. The more structured data was analysed using standard statistical methods and STATA software.

\section{RESULTS}

There were 218 patients in the Outpatient cohort. The gender division is 133 males and 85 females $(39 \%)$. The patients in the Outpatient cohort represented 24 out of 31 districts in the former West and Mid-West Development Regions. Five of the seven districts not represented are sparsely populated northern mountainous districts with few if any registered leprosy cases.

Patients from the south were somewhat under-represented in the cohort, most likely because the INF hospitals tend to draw in patients from the Hills and Mountains more than from the south where a higher percentage of patients are diagnosed by other agencies. Nationwide, at the time of the research, $80.3 \%$ of the population lived in rural areas. In the Outpatient cohort the proportion living in rural areas was $82.5 \%$. The average age of the respondents was 40.3 years, males 41.7 years and females 38.3 years.

As has been the practise in earlier published studies of leprosy delays, a cut off of six months is understood to delimit 'a short delay' while a delay in excess of six months is referred to as a 'long delay'.

The mean delay of the Outpatient cohort was found to be 28.1 months. The mean delay of males was similar to that of females (M:27.4 months, F:29.2 months). The delays of elderly versus those of a younger age were somewhat longer but failed to reach statistical significance at the $5 \%$ level. The delays by those from rural areas, however, were significantly longer than for those from cities and towns $(p<0.007)$, and so too were the delays of those from the north and the central parts compared with those from the south $(p<0.008)$.

The Patient delays from the time of the first symptom through to the start of health-seeking outside the home were lengthy. The mean Patient delay was 13.8 months, almost half of the total mean delay. The Patient delay was somewhat longer for females than for males but failed to reach statistical significance at the $5 \%$ level. Moreover, there was no significant difference between age groups, between urban and rural dwellers, or between those from districts in the south compared with those from districts further north.

According to the respondents, Patient delays were lengthy because the first symptoms were not troublesome and not perceived as serious - an observation also made in earlier studies of leprosy delays in Nepal. ${ }^{9,10}$ At $49 \%$ skin lesion or lesions were the most frequently reported 'first symptom'. In Nepal skin diseases are common and often considered an inconvenience rather than something requiring urgent treatment. There was no statistically significant difference in Patient delays between those with skin lesions as initial symptoms and others.

Self-medication and home-made treatments, when practised, were mainly in an early stage of the disease, i.e. mostly during the Patient delay, but occasionally also later. They were usually low-grade Ayurvedic treatments involving ghee massage, salves of forest products and/or efforts to balance 'hot' and 'cold'. The monetary costs tended to be low.

The Traditional Healer delay, defined as the time from first health-seeking within the magic-religious sphere (mainly shamanistic) through to the start of leprosy treatment was strongly associated with long delays. Choulagai (2005) found the same in his study in Jhapa district, in east Nepal. ${ }^{11}$ However, less than a quarter of the respondents $(22 \%)$ reported such involvements. The subject, however, is not easily explored in a short interview in a biomedical setting. Shamanic healing performances vary between quick consultations by the roadside to night-long elaborate healing sessions. Our impression is that heavy traditional healer involvements were accounted for but not minor ones. 
Most reported magic-religious health-seeking took place straight after the Patient delay but occasionally also in-between and parallel to health-seeking within the biomedical sphere. Some had been to one shaman only whereas others had gone from one to another. Monetary payments were low, but even so the total cost for gifts and paraphernalia could be heavy. Meanwhile, there was little, if any, restoration of health.

The mean cohort Biomedical delay, from first biomedical consultation to the start of leprosy treatment, was 11.6 months. There was no gender difference. However, the mean Biomedical delay of those of younger ages was less than for the elderly, and the same was true for those from urban areas versus those from rural ones, and also for patients from districts in the south compared with those from central and northern districts. So, it is mainly in connection with Biomedical delays that the earlier noted statistically significant category differences in total mean delays occurs (see above).

The national strategy is for leprosy patients in 'low endemic districts' to be diagnosed in the District hospital and for those in 'high endemic districts' to be diagnosed in the nearest public primary health centre. While some people are diagnosed in their local districts the number is less than expected. A major reason is the lack of leprosy awareness and diagnostic skills among government health service staff. This situation has been and is being worked on through various educational and training programmes with the aim of at least one leprosy competent health worker per diagnostic centre: 'the leprosy focal person'. However, there is often a high rate of absence among more highly qualified staff, while more junior staff may not have the skills needed to make a diagnosis.

The situation is further complicated by the general public often preferring private health services before public ones, and that in spite of higher costs. Government health service workers are not expected to be highly motivated and the medicines are suspected of being old or substandard. And, no less than $58 \%$ of the respondents of the Outpatient cohort reported that for 'this condition' they had not been to a public health facility, only to private onesapparently, a pattern reflecting established health-seeking habits. There tends to be some overlap of staff between public and private health services but the person providing the overlap might not be leprosy competent.

Most commonly, the first health-seeking step within the biomedical sector was a visit to a local medical hall, an over-the-counter drugs enterprise, some with staff trained for the job and some not. In the medical halls, the suggested therapy is usually according to patientreported symptom(s). Subsequent health-seeking steps tended to be to ever higher and ever more expensive medical services. In those there would be physical examinations and various tests, but often limited communication between the examiner and the one examined, and frequently without a diagnosis being spelt out, at least not to the understanding of the patient. Even so, some of the respondents testified to having been told that they suffered from an allergy, others from a nerve-related disease, yet others from uric acid in the blood, a skin disease, typhoid, and so on. Only most rarely, it seems, had the possibility of 'leprosy' been mentioned. Yet, in the end, according to the statements, nearly half of the respondents attended the INF clinic on the advice of 'a health worker' (private or public), a circumstance which indicates that 'leprosy' was suspected by the professionals more often than communicated.

Before arriving at the INF clinic, most respondents had been to several biomedical health institutions and often more than once. Time and money had been spent for consultations, $\mathrm{X}$-rays, injections, vitamins, miscellaneous drugs, and travelling. The time taken also involved a loss of income. Unfortunately, because of inconsistencies in the calculations of total cost per 
person discovered too late, the mean cost per patient is not presented. Even so, for many the expenses were more than they could afford.

In-between biomedical misdiagnoses, inappropriate treatments, and failed curative efforts, some, as mentioned, resorted to home treatments and/or to consultations with traditional healers. Meanwhile, the disease progressed.

Only one-tenth of the Outpatient cohort respondents arrived at the INF clinic with one stage of symptoms only. Instead, more than half recounted three or more symptom development stages (first there was..., then..., and after that...). Even so, the INF examination often revealed signs and symptoms not detected/reported by the patient, nerve involvements in particular. At the time of the INF examination, no less than $58 \%$ were found to be 'in Reaction' and/or with Neuritis!

According to the accounts, the respondents had rarely if ever entertained the idea of the condition possibly being 'leprosy'. And that, in spite of the fact that no less than $30 \%$ of respondents being aware of at least one relative being affected by leprosy, some with signs and symptoms similar to their own. Meanwhile, a few respondents acknowledged that they had deliberately resisted the idea of the condition possibly being leprosy.

Even at the time of the Outpatient interviews, i.e. after the initial INF examination but prior to the conclusive diagnosis, a majority (59\%) suggested that they did not suspect leprosy! According to reasons presented: no leprosy in the family; no ulcer; I do not know anything about leprosy; I do not look like a 'leper'/leprosy person' (kori/kushta rogi), and so on. Evidently, to many, 'leprosy' still conjured up a picture of missing fingers and toes, non-healing ulcers, and a deformed face-i.e. the traditional stereotype, an image out of phase with current realities. Within the Outpatient cohort 'only' $11 \%$ presented with a WHO disability Grade 2 impairment, 'a visible impairment'.

Thus, even if the presentations were voluntary they were not necessarily 'leprosy voluntary presentations' in the sense that patients presented at the INF outpatient skin clinic because they suspected leprosy. In the majority of cases, 'passive detection' might better depict the situation.

Meanwhile, some of the reasons presented by the respondents for suspecting leprosy were: an ulcer or ulcers which would not heal; no improvements in spite of several treatments; nodules on face; non-itching and/or anaesthetic skin patches.

In addition to the $11 \%$ with a visible impairment, $33 \%$ presented with at least one WHO Disability Grade 1 disability, a 'non-visible impairment'. Several, as mentioned, were 'in Reaction' and so, without the start of proper treatment then and there, a large proportion of the Outpatient cohort respondents were in absolute danger of ending up with progressive and sometimes irreversible damage and a life marred by leprosy.

Furthermore, at the time of the leprosy diagnosis, $83 \%$ of the respondents were classified as multi-bacillary (MB) cases, i.e. potentially infectious. Moreover, one-fifth (21\%) had a bacterial index of 4 or more. Thus, in many of the cases, the risk for community transmissions was and had been there.

The respondents were asked, according to their own opinion, what could have been done to shorten the delay. Most answers focused on their own and others' need to know more about early signs and symptoms and for health workers (private and public) to be more competent regarding leprosy. 'Active case finding' was not mentioned.

In late 2018, as an addendum, four leprosy patient focus groups were arranged for discussions on "reasons for long delays". In the discussions, monetary expenses were stressed and practical difficulties, such as the need to arrange for someone to accompany the patient. Carelessness of one's own health was also brought up. However, again, most suggestions 
focused on the patients' and their communities' lack of awareness and knowledge of early signs and symptoms and, as added by one of the participants: "And, health workers also do not know!"'

When a patient is diagnosed with leprosy in an INF clinic, they are then and there started on MDT. After that, according to the national strategy, the patient is expected to have their regular MDT from the nearest public health service facility. However, one of the INF hospitals has an outpatient leprosy clinic of its own and patients are given the option of referral to that clinic or to their nearest public health service centre. There were 97 patients from this hospital in the Outpatient cohort. Three-quarters of them $(n=73)$ chose the 'outpatient leprosy clinic' and that in spite of often longer and more expensive travel. A major reason for the choice was to ensure 'good treatment and no development of deformities'. Another, equally important, it seems, was 'to conceal the diagnosis'. Miller conceptualizes 'concealment' as 'self-defence' (1990:145)—a self-defence against social stigma. ${ }^{12}$

The situation of the remaining 145 Outpatient cohort respondents being referred to no less than 98 public health service facilities, reveals a wide scattering of patients in the two regions.

At the same time, as mentioned, the leprosy prevalence is far from even within the country. Thus, in the north there are some districts with hardly any registered leprosy patients, whereas some southern districts have prevalence greater than the elimination goal. It was in two of the latter, that the government massive, one-off, active case-finding operation took place in 2017.

\section{REMARKS}

The Outpatient cohort study, as pointed out in the Introduction, is one in a series of three distinct large-scale patient cohort studies within the Delay project. The plan was to include patients in diverse disease and treatment circumstances. Some were interviewed in their home environment: the Field-patient cohort $(n=100)$. Others were interviewed while hospitalized because of severe leprosy-related complications: the Inpatient cohort $(n=81)$. And, others were interviewed in the process of being diagnosed: the Outpatient cohort $(n$ = 218). The shared inclusion criteria were: a Nepali citizen from the West or the Mid-West Development Region, first time on or about to start leprosy treatment. The interviews were carried out with the help of cohort-specific open-ended questionnaires, each with a main focus on the development of symptoms and health-seeking processes. The interview data were supplemented by register information and observations, and when possible with key informant consultations. The patients in the three cohorts, with a handful of exceptions, represent voluntary presentations or passive case detections.

The Outpatient cohort mean delay of 28.1 months is similar to the mean delays of the Fieldpatient and the Inpatient cohorts, 25.3 months and 29.8 months, respectively.

The mean Patient delay of each cohort is extensive with the longest being that of the Outpatient cohort, 13.8 months. However, in none of the cohorts does the mean Patient delay exceed half of the total delay. The patient-reported main reasons for long Patient delays are basically the same: early signs and symptoms not experienced as troublesome and not recognized as indicators of something seriously wrong. In each cohort, half the respondents, or somewhat less, reported a skin lesion or lesions as the first observed symptom.

In all three cohorts, consultations with traditional healers, some taking place straight after the Patient delay and others in-between biomedical consultations, seriously extended the delays. Yet, in the Field-patient and the Inpatient cohorts as in the Outpatient cohort only a small proportion of the respondents testified to such consultations. 
In contrast, the great majority reported several visits to biomedical health service facilities prior to the leprosy diagnosis. And, in all three cohorts, as in the Outpatient cohort, the mean Biomedical delay was a year or more. In regard to health-seeking within the biomedical sphere there was a uniform pattern of private health service facilities being more frequently turned to than public ones.

Finally, in all three cohorts, according to the accounts, prior to the diagnosis, only a minor proportion of the respondents had entertained the idea of the condition possibly being leprosy.

\section{The Active case-finding operation-the Follow-up study}

In early 2017, the above mentioned large-scale case detection operation took place in the two neighbouring districts of Bardiya and Banke. The undertaking was a pilot project in line with 'the Global Leprosy Strategy 2016-2020' launched by the WHO in early 2016. ${ }^{13}$ The aim was 'early diagnosis' through active detection campaigns in high burden areas. The operation, mainly funded by the Sasakawa Memorial Health Foundation (SMHF), was organized and implemented by the Nepal Ministry of Health with the support of the WHO and two NGOs, one of the latter being INF. There was a Central Co-ordination Committee, Technical committees, Regional committees, District committees, and Municipality/village development committees.

The two NGOs, under the leadership of the District Public Health offices, were to facilitate the campaigns in one district each. For INF this meant Banke, the district in which 'INF Banke hospital' is situated.

In each district $80 \%$ of the primary health service centres were to be involved-those with most leprosy patients on treatment during the preceding three years. House-to-house surveys were to be carried out and local schools visited. Awareness of the upcoming activities was to be raised through posters, mobile megaphones, newspapers articles, and radio broadcasts. There would be Search teams, Confirmation teams, Supervisory/Technical teams, and Reporting teams.

The local campaigns were carried out in the last week of January 2017 during five parallel days in the two districts plus two extra days in Bardiya. According to the reports, a total of 282 new cases were detected.

Two monitoring missions were conducted after the campaigns, the first by SMHF staff and the second by WHO Global Leprosy Programme and Country Office staff. Some of the findings are presented in an article by Gillini et al. ${ }^{14}$ The findings indicate that $70 \%$ of the planned coverage was reached in Banke and $89 \%$ in Bardiya.

\section{METHODOLOGY}

The active case detection campaigns in the two districts presented an opportunity to extend the Delay study beyond voluntary presentations and passive detection. Thus, observations were made, documents were assembled, and key informants were interviewed. And, 15 months after the operation, and as part of the Delay project, a five-month period of field work, named 'the Follow-up', was carried out in one of the districts, the district of Banke. The focus was on local responses to the campaigns and on health-seeking processes and also on clinic attendance compliance. The latter was in view of poor compliance of those actively detected having been a major reason for the earlier change away from active case finding in the national control strategy (see above).

The district of Banke shares a border with India (map above, Figure 1). In the latest census (2011), its population was 491,000, with 39.5\% registered as having Nepali as first language, 23.7\% Awadhi, $18.5 \%$ Urdu, and $14.3 \%$ Tharu. 
In the reporting year prior to the campaigns, the official leprosy point prevalence in the district was 2.71 per 10,000 population and the new case detection rate was 34.91 per 100,000 population (196 cases). In the fiscal year of the campaigns, 2016/17, the new case detection rate jumped to 51.8 (291 cases). The year after, it was down to 28.35 (163 cases).

In Banke, 26 government health service centres, Health Posts and Primary Health Centres (PHC), functioned as hubs for the campaigns. At those, according to the records, 1328 suspect cases were examined of which 137 were diagnosed as 'new leprosy cases'. The female proportion was $54.0 \%$. The PB proportion was 59.9\%. During the preceding five fiscal years, the registered female proportion of new cases had not been above $40 \%$, and the PB proportion had not been above $50 \%$.

The members of the Search teams were engaged for three days. During the first day there were two to three hours of leprosy orientation. On the second and third days, equipped with leprosy pictorial cards, they went house-to-house mainly enquiring about hypo-pigmented and reddish skin lesions. When found, they asked about itchiness and checked for sensory loss.

The members of the Search team went two by two, ideally a man and a woman. In Banke, according to the documents, 300 female community health volunteers (FCHV) were involved. FCHVs are local women enrolled in a government programme originally initiated to promote maternal and child health. Most have only rudimentary medical training and officially they are not 'health workers' but 'volunteers'. They are mainly mobilized in vaccination schemes and the like. Even so, they are part of the local health service scene and in the campaigns they formed the backbone of the Search teams.

Persons positively screened in the house-to-house surveys were referred to the Health Post/PHC for examination by a Confirmation team. Supervisory/Technical teams stood by for problematic diagnoses.

For 'the Follow-up' study, a simple random sample of 14 of the 26 health service centres was drawn for on-site research: twelve Health Posts and two Primary Health Centres (PHC). Some were in remote areas, others in bazaar areas. Two were in all Moslem areas, two in predominantly Tharu (a Terai indigenous ethnic group) areas, a few in mainly Pahadi (people from the Hills) areas, and the rest in more diverse areas.

In 2018, during four consecutive months and one later extra month, the 14 selected health service centres were visited, each for several days. A staff group discussion was arranged at each centre, as were two individual staff interviews. Local reports, referral slips, and patient files were studied and discussed. Also at each location, two volunteers from the Search team were separately interviewed, some in their homes and some in other places. Moreover, in each catchment area, when feasible, at least two 'campaign-detected new cases' were interviewed.

The main instrument for the patient interviews, an open-ended questionnaire, was basically the same as the one used for the Outpatient cohort (see above) with some additional questions. The interviews were arranged and carried out by the same interviewer, a senior INF leprosy officer well acquainted with the Terai and its major languages. None of those approached declined participation.

THE FOLLOW-UP RESULTS AND REMARKS

The number of new cases detected within the Follow-up sample during the campaigns varied from none to 15 per centre. In two of the catchment areas no 'new case' was detected. In the remaining twelve, according to the lists, there were 75 'new case detections' (slightly more than half of the Banke 'new detections'). The female proportion was $62 \%$ and the PB proportion, $69 \%$. 
One of the centres with no new detection is a small Health Post with a history of few leprosy patients. The other is a busy PHC next to a town. There were 20 Search units at work within its catchment area. No specific difficulties were encountered. Even so, only 40 'suspect cases' were identified. Stated reasons as to why there were so few positively screened cases included "Perhaps they did not want to be found" and "The Survey teams worked from 8 am to 5 pm and that is a busy time and most likely some people were not at home." All the positively screened persons showed up at the PHC for examination, but none was diagnosed as a leprosy case. The latter was explained by part of the catchment area being a town area and town people are aware of various treatment possibilities and do not wait around for a campaign. Moreover, the INF hospital, it was said, is well known and easy to reach, and this is where most of the PHCs' leprosy patients are diagnosed, not in the PHC.

A busy Health Post in a predominately Tharu area on the outskirts of a town had the highest number of newly detected cases in the Follow-up sample: twelve diagnosed at the Health Post and three at INF Banke hospital. Of the 15 new detections, 12 were females. The acting Health post in-charge, a female, said that there had been a male and a female in 19 of the 20 Survey units. The main reason for the unbalanced male/female ratio among the new detected cases, she suggested, was because men seek and get help earlier than women, they are more mobile and, in contrast to women, they do not need someone to accompany them. Moreover, a woman with skin lesions only is likely to postpone health-seeking and most of the female cases detected had skin lesions only. In addition, the surveys took place during working hours, which is when most men were out of the house. She was not surprised to learn that not only in 'her' Health Post but in the Banke campaigns as a whole, more female than male patients had been detected, and more PB than MB cases.

The interviewed health workers $(n=28)$ expressed their satisfaction with the campaigns but stressed the need for recurrent surveys as most likely some patients had not been reached. And, because of frequent movements across the border into highly leprosy endemic areas in northern India there would inevitably be further cases. They also emphasized the need for more leprosy training for themselves, in part because of frequent staff transfers.

The FCHVs interviewed $(n=28)$, spoke highly of the leprosy orientation and the campaigns and declared that they were more than willing to participate in future leprosy surveys. The task, they said, was particularly suitable for females because it did not take them outside their own areas and women preferred to be contacted and examined by women. Moreover, people knew them. They said that they had not encountered any problems as most people knew that 'a skin disease campaign' was to take place. 'Leprosy', they said, was rarely mentioned. In certain circumstances, however, suspect cases were told that their condition "might be leprosy, or early leprosy". Meanwhile, in the schools, students with suspect signs were encouraged to make their way to the Health Post or PHC for "a closer examination of the skin".

According to the instructions, persons affected by leprosy should participate in the campaigns whenever possible. However, on the ground, the latter was rarely realized, at least not in the health service facilities of the Follow-up sample. Thus, in only one of them was a person affected by leprosy mobilized. In that one and only place, two persons affected by leprosy were part of the Search team, one of them a local community leader. In one of the other places, a person affected by leprosy was used to demonstrate the kind of signs and symptoms to look out for. But that was it. The main reason presented was a preference not to share the financial incentives with others and so they were simply not asked to participate.

In their article, Gillini et al. ${ }^{14}$ write that health workers mentioned geographical constraints and social stigma in the community as possible reasons for positively screened referrals not 
seeking diagnostic confirmation (p. 81). In the Follow-up sample, the interviewed health workers and volunteers agreed to the possibility of stigma as a deterrent, but not as a major one. The reason supposedly being that in the Terai, in contrast to other parts of the country, leprosy stigma is no longer a major problem. However, this, it was said, did not apply for those with visible signs and symptoms, and for unmarried young females. Some of the thoughts presented included statements such as "Before, it was looked upon as a curse but not necessarily so today, particularly not if only a skin lesion"; "When others know-others dominate"; "Worse for women, some are not allowed to cook"; "Other people keep a distance, perhaps even after the signs are gone"; "Those diagnosed do not want others to know".

Persons positively screened in the house-to-house surveys were referred to the Health Post/PHC for examination by the Confirmation team. In the case of leprosy, the person was taken to the side and informed that it was kushta rog (leprosy) and that leprosy is a disease among other diseases, a skin and nerve disease caused by kitaunu (bacteria/minute animals), curable by medicines, and if treated early there will be no deformity. They were started on MDT, given a four-week MDT blister pack, and told to return for the next one. Most 'difficult to diagnose' cases were referred to the INF Banke hospital and so were those with clinical signs suggesting a possible need for more than 12 months of MDT.

According to the FCHVs interviewed, only most rarely had any of those referred for an examination not proceeded to the health service facility. Even so, one or two knew of someone who had chosen to go straight to the INF hospital: "most likely to avoid being shamed". After the campaigns, only one of the FCHVs interviewed had 'referred' a suspect case and not to the nearest Health Post but to the INF hospital.

Three of the 75 listed new detected cases in the Follow-up sample were patients who had previously been on MDT. Of the remaining 72, a convenience sample of 31 cases (12 males, 19 females), representing twelve catchment areas, were interviewed by help of an open-ended questionnaire. The age range (at diagnosis) was 10-75 years with a mean age of 35.7 years.

Thirty persons were interviewed in their homes and one over the phone (out of the area). None of them expressed negative feelings about the campaigns but rather gratefulness for having been found. They all knew the name of the disease ("kushta'/"kushta rog'). However, apparently, some were not sure of why their condition was 'leprosy'. Even so, the diagnosis had seemingly been accepted.

In spite of the leprosy information supposedly provided at diagnosis, somewhat less than half said that they did not know the cause of leprosy and/or of their own condition. Several presented more than one idea. Some of the ideas suggested were: physical weakness; a lack of blood; all things happen because of Allah; from dirty water; from food leftover (Jutho); having worked in dirty places; your own body; a genetic disease. At the same time, most of them knew that leprosy is a transmittable disease, although the mechanisms of transmissions were not well understood. 'Bacteria' (kitaunu) was not mentioned.

Only two of the 31 respondents acknowledged that they had suspected leprosy prior to the campaign and yet, one third testified to having/having had a family member and/or a close relative affected by the disease. However, signs and symptoms had not necessarily been the same and when not identical a possible connection had evidently been missed. Moreover, for some, the leprosy of a relative was something they had heard about but not 'seen' themselves. Furthermore, in the minds of most, 'leprosy' meant 'deformity', and most of those interviewed within the Follow-up sample did not have a leprosy-related deformity (see below). 
So, what about the clinic attendance compliance? Twenty-nine of the 31 in the interview cohort completed their treatments according to schedule. Even a man in jail (illegal woodcutting) did so by arranging for his wife to pick up his monthly blister pack and have it delivered to him. One of the two who did not complete the treatment as per schedule was a young woman who said that she discontinued the therapy after the first batch of medicines because suddenly there was ' jhum jhum' (tingling/throbbing feelings) in her hands and feet. The other person who dropped out was also a young woman, an MB case. After seven months of regularly having picked up her blister packs she left the area for her maita (parental home) and in connection with that the therapy was discontinued. She was the one interviewed over the phone.

To broaden the sample, the compliance of those in the Follow-up sample not included in the interview cohort $(n=41)$ was investigated. According to the local notifications, the great majority were regular in attendance with 35 'released from treatment' (RFT) within the prescribed period. Six had been written off as 'defaulted'. One of the latter was a woman known to have moved to her son in America and another was a woman, whose husband was in the police who had left the area in connection with her husband being transferred. We met with two of the remaining four. One of them, a rickshaw driver, lived partly in India, partly in Nepal. He had left for India shortly after the diagnosis but had continued his MDT treatment in India-a fact corroborated by previous skin lesions having disappeared. The other was a young woman, who said that monkeys had taken her patient card and her medicines (the family said the same). At the time, there was some jhum jhum in her hands, but her single skin lesion was gone and thus she had not bothered to return to the Health Post. The remaining two, were registered at the same Health Post. After the first supervisory dose provided at the time of the diagnosis, none of them had reappeared and there had been no follow-up. No one seemed to know what had happened with them. The Health Post in-charge finally suggested that most likely they had opted out because they had not fully accepted the diagnosis or because they were Indians and had left Nepal.

Thus, according to local notifications, eight of a total of 72 patients in the Follow-up sample had discontinued their treatments, yet for very different reasons. At the same time, the great majority, $89 \%$, had completed their therapy. Even though the sample is limited, it exemplifies a rather high rate of compliance, i.e. not a big drop out.

We learnt that among those interviewed only half of the RFT patients suggested that they were 'cured' with the other half suggesting that they were not or most likely not. The main reason presented for the latter was that symptoms had not disappeared or had not disappeared fully, for instance: jhum jhum; sensory loss; skin patches: pain; and a clawed hand. The reverse was true for those who suggested that they were cured. They were cured, they said, because of the disappearance or near disappearance of previous symptoms. The patient-understanding of the Confirmation teams' message of leprosy being curable by medicines (a long-standing official mantra) had obviously not been understood as intended-in the biomedical sense, no more viable $M$. leprae. Instead, the understanding and interpretations were linked to what was felt and seen.

In the campaigns, in connection with the leprosy diagnosis, efforts were made to limit the number of other individuals who became aware of the diagnosis. The great majority of those interviewed testified to wanting to conceal. Even so, in all cases except two, others were in the know. The give-away, it was said, was mainly in connection with the diagnosis. One of those interviewed counteracted rumours by declaring her condition to be diabetes and another by proclaiming that his condition had been diagnosed as daad, fungus. An unmarried girl in her later teens was particularly worried. She said that everyone knew about her leprosy even 
though there had only been one single skin patch which was now gone. She told us that in her village there was a girl slightly older than herself with patchy skin colouring (vitiligo) who had not got married and that in spite of her father having offered money for someone to marry her. The villagers, she added, said that the other girl had 'white leprosy'. Meanwhile, she herself feared that her fate would be the same. In general, however, at the time of the interviews, approximately a year and an half after the diagnosis, most respondents seemingly were not all that worried about others having learnt about their condition, particularly as there was not much there for anyone to see.

The mean delay of the interview cohort was 23.7 months (see below). The mean Patient delay was 9.2 months, again less than half the mean delay. However, the sample is limited and reported Patient delays varied from 1 to 36 months with approximately half above and half below six months.

As in the other cohorts, the most frequently reported 'first symptom' was a skin lesion. The lesions were mostly assumed to be fungus (daad), a commonplace phenomenon in the hot and humid Terai and not perceived as a matter of urgency. At the same time, there were and had been a variety of self-diagnoses: the result of an insect bite; an allergy, and so on.

According to the narratives, during the Patient delays, somewhat less than half had done nothing or next to nothing, whereas somewhat more than half had tried self-medication and home treatments. The latter, as in the other cohorts, were mainly in the form of lowgrade Ayurvedic attempts, mostly applications of self-produced herbal salves and ointments, sometimes supplemented by Humoral treatment efforts to balance 'hot' and 'cold'.

'For this condition', according to the accounts, few had turned to 'traditional healers', to the magic-religious sector. Nowadays in the Terai, it was said, traditional healers are mainly consulted for sudden illnesses and when children are sick. Meanwhile, the uptake of biomedical health services had redoubled.

Even so, prior to the campaigns, according to the accounts, for 'this condition' slightly less than half had not been to a biomedical health service facility.

Meanwhile, among those who had been to a biomedical health service facility the Biomedical delays varied from 1 to 93 months: a wide range within a limited sample. Some had been to only one health centre whereas others had been to several, yet rarely to the extent common in the other cohorts. Even so, the same pattern of public health services being side-stepped for private ones was there, and highly so. Thus, in the Follow-up interview cohort only three respondents testified to having turned to a public health service facility 'for this condition', prior to the campaigns, and none of them to the nearest Health Post/PHC but to the government Zonal hospital in the district capital.

Two of the respondents reported exceptionally long total delays from first symptom to the start of MDT. However, the initial symptoms of the one with the longest total delay (180 months) may not have been leprosy related and thus his delay is neither included in the mean delay of the interview cohort nor in the mean Patient delay. The person with the second longest delay (94 months), a woman, had been taken to the government Zonal hospital a month and a half after the first observed symptom, a patch with sensory loss with a blister. However, leprosy was not diagnosed. Over the years, there were more signs and symptoms, including recurring ulcers, but no further health-seeking outside the home. During the campaigns not only she but also her husband was found to be leprosy-affected, her husband's symptom having appeared eight months earlier.

Having excluded the reported longest delay (see above), the mean delay of those formally interviewed was 23.7 months with $74 \%$ having reported delays of more than six months, $58 \%$ 
more than twelve months, and $23 \%$ more than 24 months. Thus, most delays clearly fall into the category of 'long delays' (see above).

To broaden the sample size, the delay notifications in the patient files of those within the Follow-up sample but outside the interview cohort $(n=41)$ were reviewed. Three files were not found and five did not have the necessary information. The mean registered delay of the remaining 33 patients was 22.8 months with $67 \%$ being delays of more than six months.

Thus, timewise most of the new detected cases within the Follow-up sample were not "early diagnoses' but late ones!

Only two of those formally interviewed reported 'one symptom development stage' (first there was..., then..., and after that...) only. Even so, the reported developments of signs and symptoms were less than in the other cohorts. And, at the time of the diagnosis, according to the patient files, $74 \%$ were without a WHO disability grade 1 or 2 impairment. For the Outpatient cohort, the corresponding figure was $56 \%$.

To enlarge the sample-base, the patient files of those not interviewed within the Follow-up sample $(n=41)$ were also screened for recorded disability grade at diagnosis. According to the 38 files at hand, again 74\% were without a WHO disability grade 1 or 2 impairment.

In addition, according to the patient files, and most likely of crucial importance for less active health-seeking, at the time of the diagnosis, only one in the interviewed sample and one only among those not interviewed within the Follow-up sample showed signs of being 'in Reaction'.

In the Follow-up sample as a whole, $66.7 \%$ were classified as PB leprosy, i.e. with the less advanced type of the disease, lower infectiousness included. Had the PB cases not been found and started on treatment some of them might have self-healed but most likely a larger proportion would have progressed to MB leprosy with amplified negative consequences for the individual and for the community.

\section{Remarks and discussion}

The aim of the project was to explore patterns of health-seeking and reasons for long leprosy delays. Whereas the Outpatient cohort includes patients from most parts of the West and Mid-West Development Regions, the Follow-up sample represents patients from one district only. And, whereas the Outpatient cohort includes patients from high and low leprosy burden districts, the Follow-up sample includes patients from a high burden district only. Moreover, whereas the Outpatient cohort contains a large number of respondents $(n=218)$, the Followup sample $(n=75)$ is considerably smaller. Thus, the two cohorts are clearly very different, but even so in regard to the main theme they are complementary-the former representing voluntary presentations or passive detections whereas the latter represents active case detection in the field.

The high number of cases detected in the Active case detection operation is remarkable and even more so as in Banke, according to Gillini et al. (see above), only $70 \%$ of the planned coverage of $80 \%$ was realized. At the same time, the high number of new detected cases in the campaigns runs parallel to the two surveyed districts being high burden districts. Even so, the high number testifies to voluntary presentations and passive detection not being sufficient if Nepal is to free itself of leprosy. An observation and conclusion also made by Gillini et al."The Nepal experience”, they write, “... highlights the fact that many cases of leprosy are still being 'missed' under passive detection modalities ..." (p. 82).

The mean delays of the Outpatient and the Follow-up cohorts were both long, 28.1 months and 23.2 months respectively. The Outpatient cohort's long mean delay was not a surprise as 
it is similar to the mean delays of the previously studied Field-patient and Inpatient cohorts. However, the long mean delay of those in the Follow-up sample is surprising, particularly bearing in mind that the respondents were residents of a district with numerous health service facilities, an INF hospital included.

The information obtained suggests that compared to the Outpatient sample and prior to diagnosis, there had been a slower progression of the disease and ill health in the Follow-up sample and thus less of an urge to seek qualified help. Thus, $43 \%$ of the respondents in the Outpatient sample presented with WHO Grade 1 and/or Grade 2 disabilities compared with $26 \%$ in the Follow-up sample. And, the respondents of the Outpatient cohort reported more symptom development stages than those in the Follow-up sample. And, whereas a sizeable proportion of the Outpatient respondents were 'in Reaction' at the time of the diagnosis, this was the case for only two of the respondents in the Follow-up sample.

The lower proportions of lived-in sickness in the Follow-up sample are most likely linked to its high percentage of PB cases, $67 \%$ - a considerably higher proportion than for the nation as a whole as well as for the Outpatient cohort, $27 \%$.

Significant is also the high female proportion, 54\%, of those detected in the Active case operation-a percentage considerably higher than for the nation as a whole as well as for the Outpatient cohort, $39 \%$.

Three-quarters (74\%) of the new detected cases in the Active case detection campaigns were not 'early diagnoses' (the WHO strategy) yet they were all 'earlier diagnoses' than they would have been had the active case detection not taken place - an important outcome in itself.

The major reasons for the long Patient delays reported in both cohorts is that early symptoms were not troublesome and so not recognized as signs of a possibly dangerous conditiondefinitely not as signs of leprosy. The latter, it seems, is closely connected with traditional ideas and images of leprosy-ideas and images out of touch with the present reality and with the biomedical definition of leprosy.

In both cohorts, less respondents than expected testified to consultations with traditional healers. However, in both cohorts there might have been some under-reporting as most Nepali people are aware of magic-religious treatments not being highly thought of in biomedical circles. At the same time, the gradual development of symptoms, not a sudden onset, might be part of the explanation for less than expected consultations with shamanistic healers. Another likely explanation is the ever greater availability of biomedical services in most parts of the country.

While most respondents in the Outpatient cohort had been to several biomedical health institutions prior to the leprosy diagnosis only half of the respondents in the interview cohort of the Follow-up sample reported such a visit, a circumstance most likely linked to the above mentioned variance in experiences of ill health. Even so, among those who had been to a biomedical facility, the pattern of public health services frequently being side-stepped for private health services was there.

Many of the Outpatient cohort respondents testified to high costs for their health-seeking prior to the leprosy diagnosis-high costs ill afforded by most. Gillini et al. report that the total expenditure for the Active case detection operation amounted to US\$120,000. Expenditures equal to a cost of US\$424 per new patient or US\$534 per 'additional' case- “"a relatively high cost" it was noted (p. 81).

By most measures the active case detection campaigns were a success and they were followed by good clinic attendance adherence and completed treatments. However, it was 
a one-off operation and, as voiced by interviewed health workers and by Gillini et al., in a leprosy situation like that in Banke and Bardiya, a one-off operation is not enough.

The findings from both the Outpatient Cohort and the Follow-up Study reinforce concerns about lengthy delays and the impact on the individual affected and the longer the delay the greater the opportunity for transmission.

\section{Conclusions}

The findings of the Delay project show that despite great achievements in leprosy care and control over the years, leprosy delays are still long, too long. And thus, despite the national elimination goal having been reached, additional efforts are essential if Nepal is to free itself of leprosy.

The high number of newly detected cases in the active case detection operation highlights the fact that in high burden districts, and most likely also in other districts, voluntary presentations and passive case detection is not sufficient. Thus, a double approach of selfreferral and active case detection is likely to be needed. For the former to work well awarenessraising on early signs and symptoms at the primary level is necessary, and as patients and patients-to-be are widely scattered, a broad-scale dissemination of information is called for, some realized through wide-reaching means such as radio and other relevant social media, some through tailor-made material and inputs for decision makers and local units/groups such as 'mothers groups' and faith-based organizations. Moreover, the leprosy skills of professionals and volunteers within the health care sector (private, public, and traditional) have to be upgraded and sustained at least to the degree that leprosy-related early signs and symptoms trigger the right response: timely diagnosis, with referral where appropriate. Special attention should be paid to private medical halls as it is to such places that most patients turn in the early stages of their health-seeking outside the home. In these, as well as in other health service facilities, well-informed and well-placed information posters can serve as repeated reminders to staff as well as to visitors about leprosy still being endemic. Active case finding does not necessarily have to be in the form of centrally decided, costly, massive surveys but on a more moderate and local scale such as 'contact examinations' and school screenings. Some such activities might be part of other public health efforts such as immunization programmes. The potential for a more active role of FCHVs in control as well as in leprosy care should be investigated as should an appropriate involvement of persons with leprosy experiences of their own - the latter, in view of 'Leprosy' not being a condition of body and bacteria only but of lives.

\section{Acknowledgements}

The authors want to express sincere thanks to all patients and INF leprosy staff involved in the project. We also want to thank the Leprosy Research Initiative, the Netherlands, for their generous financial support, and the Nepal Health Research Council for ethical approval.

\section{References}

1 Hyland J. A socio-cultural study of leprosy in Nepal. Ph.D. Thesis, University of Tasmania, Australia. 1993.

2 Jain MC. Leprosy scenario in Nepal. J Nepal Med Assoc, 2008; 47(172): 250-263.

3 Leprosy Control Programme Annual Report: 2074/75 (2017/18). Ministry of Health \& Population, Government of Nepal, Teku, Kathmandu, Nepal.

4 Engelbrektsson U-B, Subedi M. Stigma and leprosy presentations, Nepal. Lepr Rev, 2018; 89: 1-8. 
5 Engelbrektsson U-B, Subedi M, Nicholls P. The challenge of health-seeking: recollections of leprosy inpatients in post-elimination Nepal. Lepr Rev, 2019; 90: 1-11.

6 Subedi M, Engelbrektsson U-B. Factors contributing to delay in diagnosis and start of treatment of leprosy: Analysis of help-seeking. Narratives from a community study in Dang district. Dhaul J Sociol Anthropol, 2018; 12: 11-17.

7 Subedi M. Medical Anthropology of Nepal. Kathmandu, Nepal: Udaya Books Ltd., 2001.

8 Nicholls PG, Croft RP, Richardus SG, Smith WSC. Delay in presentation, an indicator for nerve function status at registration and for treatment outcome - The experience of the Bangladesh acute nerve damage study cohort. Lepr Rev, 2003; 74: 349-356.

9 Heijnders ML. Experiencing leprosy: perceiving and coping with leprosy and its treatment. A qualitative study conducted in Nepal. Lepr Rev, 2004; 75: 327-337.

10 Choulagai BP. Patient delay in leprosy treatment in Jhapa district, Nepal. Master Thesis, Tribhuvan University, Kathmandu, Nepal. 2005.

11 Choulagai B, Onta S, Phattarai P. Patient delay in leprosy treatment in Jhapa district Nepal. J Nepal Health Res Council, 2005; 3: 29-34.

12 Miller C. Decision Making in Village Nepal. Kathmandu: Pilgrims Publishing, 2000.

13 WHO. Global Leprosy Strategy 2016-2020. Accelerating Towards a Leprosy-Free World. Geneva: World Health Organization; p. 216.

14 Gillini L, Cooreman E, Pandey B et al. Implementing the global leprosy strategy 2016-2020 in Nepal: lessons learnt from active case detection campaigns. Lepr Rev, 2018; 89: 77-82. 